\title{
Diagnóstico pouco frequente de dor abdominal em unidade de emergência infantil
}

\author{
Unusual diagnosis of abdominal pain in pediatric emergency unit
}

\author{
Suelen Bianca S. Martins ${ }^{1}$, Maria Ângela B. Brandão ${ }^{2}$, Marcelo Barciela Brandão ${ }^{3}$, Marcelo Conrado dos Reis ${ }^{4}$, \\ Maria de Fátima C. P. Servidone ${ }^{5}$, Mariana Porto Zambon 6
}

\section{RESUMO}

Objetivo: Relatar um caso de doença péptica na infância em escolar, com queixa inicial de dor abdominal aguda e palidez.

Descrição do caso: Escolar de nove anos procurou pronto-socorro infantil com queixa de dor abdominal súbita e palidez. Não apresentava outros sintomas ou sinais ao exame físico além de mucosas descoradas e hemograma com nível baixo de hemoglobina. Durante a observação intra-hospitalar, apresentou episódio de melena. Foi realizada endoscopia digestiva alta, sendo observadas gastrite erosiva de antro e úlcera duodenal, com Helicobacter pylori positivo. O paciente foi tratado com esquema tríplice (inibidor de bomba de prótons e dois antimicrobianos) por uma semana e, posteriormente, acompanhado ambulatorialmente.

Comentários: $\mathrm{O}$ caso em questão descreve uma doença rara em unidade de emergência pediátrica. O paciente referia dor abdominal e observou-se palidez confirmada por baixo nível de hemoglobina, que se manteve nos exames subsequentes durante a observação no pronto-socorro infantil, dificultando o diagnóstico de sangramento ativo. Porém, a presença de melena na evolução facilitou e propiciou a condução do caso para se chegar à hipótese de sangramento digestivo alto. Realizada endoscopia digestiva alta, que confirmou o diagnóstico de gastrite erosiva de antro e úlcera duodenal $H$. pylori positiva, sendo, então, necessário tratamento específico, inclusive para erradicação da bactéria.

Palavras-chave: dor abdominal; úlcera péptica; Helicobacter pylori.

\section{ABSTRACT}

Objective: To report a case of peptic disease in a school child whose first symptoms were acute abdominal pain and paleness.

Case description: A nine-year-old school child presented to a pediatric emergency unit with acute abdominal pain and sudden pallor. There were no other symptoms or signs on physical examination, except for discolored mucous and a low hemoglobin level. During hospital observation he presented one episode of melena. The esophagogastroduodenoscopy showed erosive antrum gastritis and duodenal ulcer, with Helicobacter pylori. He was treated with inhibitor pump proton and two antimicrobial for two weeks.

Comments: This is a rare presentation in the pediatric emergency unit. The patient had abdominal pain and pallor confirmed by low hemoglobin level, which remained in subsequent blood tests, delaying the diagnosis of active bleeding. The occurrence of melena during hospitalization led to the hypotheses of high digestive bleeding. An esophagogastroduodenoscopy was done and confirmed the
Instituição: Departamento de Pediatria da Faculdade de Ciências Médicas da Universidade Estadual de Campinas (Unicamp), Campinas, SP, Brasil ${ }^{1}$ Residente da Faculdade de Ciências Médicas da Unicamp, Campinas, SP, Brasil

${ }^{2}$ Médica Assistente do Ambulatório de Gastroenterologia e Endoscopia Digestiva Pediátrica do Hospital de Clínicas da Unicamp, Campinas, SP, Brasil

${ }^{3}$ Médico Assistente da Unidade de Urgência Referenciada Pediátrica e da Unidade de Terapia Intensiva Pediátrica do Hospital de Clínicas da Unicamp, Campinas, SP, Brasil

${ }^{4}$ Responsável e Médico Assistente da Unidade de Urgência Referenciada Pediátrica do Hospital de Clínicas da Unicamp, Campinas, SP, Brasil ${ }^{5}$ Médica Assistente do Ambulatório de Gastroenterologia e Endoscopia Digestiva Pediátrica do Hospital de Clínicas da Unicamp, Campinas, SP, Brasil
${ }^{6}$ Doutora; Professora Assistente do Departamento de Pediatria da Faculdade de Ciências Médicas da Unicamp; Médica da Unidade de Urgência Referenciada Pediátrica do Hospital de Clínicas da Unicamp, Campinas, $\mathrm{SP}$, Brasil

Endereço de correspondência:

Suelen Bianca S. Martins

Rua Izabel Negrão Bertotti, 141, apto. 13 - Primavera

CEP 13078-508 - Campinas/SP

E-mail: suelen_martins@hotmail.com

Conflito de interesses: nada a declarar

Recebido em: 30/3/09

Aprovado em: 4/7/09 
presence of erosive antrum gastritis, duodenal ulcer and $H$. pylori infection. The treatment of the patient included bacterial eradication.

Key-words: abdominal pain; peptic ulcer; Helicobacter pylori.

\section{Introdução}

A dor é uma das manifestações mais precoces da enfermidade ou da lesão tecidual ${ }^{(1)}$. Dor abdominal aguda é um problema comum em crianças e constitui um diagnóstico difícil para o pediatra ${ }^{(2)}$. Numerosos distúrbios podem causar dor abdominal, sendo as causas mais comuns a gastroenterite e a apendicite ${ }^{(3)}$. Embora muitos casos de dor abdominal aguda sejam benignos, alguns requerem diagnóstico e tratamento rápidos para minimizar a morbidade, principalmente quando recebidos em unidade de emergência ${ }^{(2)}$.

A avaliação do paciente com dor abdominal aguda utiliza como instrumento a história, que valoriza dados referentes à dor, fatores associados à presença de vômitos, alteração do hábito intestinal e antecedentes mórbidos pessoais. A idade é um fator chave para avaliação da causa, pois a incidência e os sintomas das diferentes doenças variam de forma significativa de acordo com a faixa etária ${ }^{(2)}$. Além disso, a dor abdominal apresenta-se como um sintoma de difícil caracterização, principalmente em lactentes e crianças pequenas, já que depende dos dados fornecidos pelos pais que são influenciados pela sua capacidade de observação e grau de angústia ${ }^{(2)}$. O exame físico completo e meticuloso deve ser realizado para permitir um diagnóstico preciso e afastar os principais diagnósticos diferenciais ${ }^{(1)}$.

A seguir, é relatado um caso de dor abdominal aguda associado à palidez atendido em pronto-socorro infantil (PSI).

\section{Descrição do caso}

Paciente do sexo masculino, nove anos de idade, procurou PSI com queixa de dor abdominal e palidez iniciadas algumas horas antes. A dor estava localizada em região periumbilical, tinha forte intensidade e era acompanhada de náusea sem vômito e palidez. Negava febre, parada da eliminação de gases e fezes, alteração urinária ou intestinal. Negava também o uso recente de medicamentos ou episódios anteriores semelhantes. Relatava antecedente de anemia com um ano de idade tratada com sulfato ferroso e irmã de três anos em tratamento de anemia ferropriva. Ao exame de entrada, apresentava-se descorado 3+/4+, abdome plano, flácido, levemente doloroso à palpação, ruídos hidroaéreos presentes, descompressão brusca negativa e extremidades frias.

$\mathrm{Na}$ investigação inicial, foram solicitados os seguintes exames: hemograma (HMG), exame de sedimento urinário (EAS), amilase, gasometria com eletrólitos, radiografia de tórax e abdome. À admissão, o hemograma mostrou hemoglobia de $7,5 \mathrm{~g} / \mathrm{dL}$, hematócrito de $22 \%$, com volume corpuscular médio de $78,4 \mathrm{fl}$, plaquetas de $337.000 / \mathrm{mm}^{3}$ e leucócitos de $10.700 / \mathrm{mm}^{3}$. Tais valores não se modificaram após 12 horas. A urina I mostrava densidade de 1030, $\mathrm{pH}$ de 7,0, 1 hemácia e 1 leucócito por campo. O coagulograma era normal e a tiragem sanguínea $\mathrm{O}$ positivo. A gasometria arterial revelava $\mathrm{pH}$ de 7,39 e $\mathrm{HCO}_{3}$ de $22,2 \mathrm{mEq} / \mathrm{L}$. A glicose era $103 \mathrm{mg} / \mathrm{dL}$ à admissão, o sódio $135 \mathrm{mEq} / \mathrm{L}$, o potássio $3,9 \mathrm{mEq} / \mathrm{L}$, o cloro $108 \mathrm{mEq} / \mathrm{L}$ o cálcio $1,22 \mathrm{mg} / \mathrm{dL}$ e a amilase $73 \mathrm{U} / \mathrm{L}$, sendo o valor de referência para a última abaixo de 100U/L. Assim, os exames laboratoriais mostraram-se normais, exceto por uma anemia importante, normocítica e normocrômica, compatível com o exame físico. Os exames radiológicos de tórax e abdome também apresentaram-se sem alterações.

O paciente evoluiu bem, com melhora da dor abdominal, porém mantendo palidez. Os exames de controle, hemoglobina/hematócrito $(\mathrm{Hb} / \mathrm{Ht})$, certificavam que não havia sangramento ativo ou perda sanguínea, não sendo necessária a administração de concentrado de hemácias. Nesse momento, apresentou fezes enegrecidas e fétidas, sugerindo melena. Foi realizado toque retal, no qual não foram encontradas alterações nem sinais de sangramento ativo.

Foi solicitada então uma endoscopia digestiva alta (EDA) para pesquisar a possível causa do sangramento digestivo alto. O exame resultou em gastrite endoscópica erosiva leve de antro (erosões elevadas recobertas por fibrina), sem sangramento ativo, e úlcera duodenal ativa (Sakita A) e classificação de Forrest III (presença de coágulo sentinela, sem sangramento ativo, com base da úlcera limpa). Realizou-se biópsia que resultou em gastrite crônica moderada, com atividade leve em mucosa do tipo antro/piloro. A pesquisa para Helicobacter pylori foi positiva.

O paciente recebeu alta do PSI com tratamento para úlcera duodenal $H$. pylori positiva usando esquema tríplice: inibidor de bomba de prótons (IBP) - omeprazol - associado a dois antimicrobianos (amoxicilina e claritromicina) por uma semana. Foi encaminhado para seguimento ambulatorial. Após quatro meses do início do tratamento, foi realizada nova EDA, que mostrou úlcera cicatrizada (Sakita $S$ ) e $H$. pylori positivo. $\mathrm{O}$ resultado foi considerado reinfecção pela 
equipe da gastroenterologia pediátrica, sendo prescrito novo tratamento com omeprazol, amoxicilina e metronidazol.

O presente relato foi submetido ao Comitê de Ética da Faculdade de Ciências Médicas da Universidade Estadual de Campinas (Unicamp) e aprovado para publicação.

\section{Discussão}

A dor abdominal aguda como queixa em Serviço de Emergência Pediátrica remete às causas mais comuns: gastrenterite e apendicite. Porém, não podem ser desconsideradas vários outros diagnósticos diferenciais. Dentre as causas clínicas, pode-se citar adenite mesentérica, crise falciforme, parasitose intestinal, pancreatite, cetoacidose diabética e infecção do trato urinário. Dentre as causas cirúrgicas de dor abdominal, destacam-se: divertículo de Meckel, hérnia encarcerada, úlcera péptica perfurada, bridas e invaginação intestinal entre outras.

No caso relatado, o paciente não apresentava sintomas de gastrenterite nem quadro inicial compatível com apendicite. Queixava-se de dor abdominal inespecífica, sem alterações nos exames iniciais, exceto por anemia importante, que chamava atenção ao exame físico, fazendo parte inclusive da queixa inicial. Somando-se os sintomas iniciais ao quadro de melena no PSI, chegou-se à hipótese diagnóstica de sangramento digestivo alto e doença péptica por úlcera duodenal.

A doença péptica é definida por doença ulcerosa e nãoulcerosa, causada por secreção cloridopéptica. Pode ocorrer no terço inferior do esôfago, estômago, duodeno proximal e no divertículo de Meckel. É uma doença comum em adultos e predominante no sexo masculino, porém desconhece-se sua real incidência em crianças. Observa-se um aumento no número de casos desde a disponibilização de técnicas endoscópicas seguras para o diagnóstico nessa faixa etária ${ }^{(4)}$. A prevalência em grandes centros pediátricos é de quatro a sete novos casos por ano ${ }^{(5)}$.

Classifica-se a úlcera péptica, de acordo com a localização, em gástrica, quando há redução dos fatores protetores (muco, bicarbonato ou prostaglandinas), e/ou duodenal, quando há aumento dos fatores agressivos (secreção ácida e pepsina). Outra maneira de classificar segue a provável etiologia: primária e secundária. A primária ocorre na ausência de doença sistêmica subjacente, é mais frequente no bulbo duodenal, em escolares e tem curso clínico crônico. A secundária está associada à doença aguda ou crônica, mais frequente no estômago e/ou duodeno, acometendo neonatos, lactentes e pré-escolares, com curso clínico mais agudo ${ }^{(6)}$. Geralmente a lesão é única, arredondada ou ovalada, com base composta por tecido de granulação, plana, regular e coberta por exsudato fibrinoide branco ou branco-acizentado. $\mathrm{O}$ aspecto endoscópico da úlcera depende do momento em que é observada, de acordo com o ciclo vital descrito por Sakita ${ }^{(7)}$ : ativa - A, em cicatrização - H, e cicatrizada - S. No caso descrito, a úlcera foi classificada como duodenal, primária e ativa.

O conceito fisiopatológico é de que a úlcera resulte da ruptura do equilíbrio entre os fatores agressivos e protetores da mucosa gastroduodenal ${ }^{(4)}$. Sua etiopatogenia está associada a fatores genéticos, distúrbios fisiológicos ainda não bem estabelecidos e a fatores ambientais como tabagismo, uso de medicamentos e infecciosos. Nessa última categoria, destacase como importante fator causal a bactéria $H$. pylori $i^{(4)}$. A evidência definitiva da sua participação como fator etiológico é de que o tratamento com antimicrobianos altera substancialmente a evolução da doença ${ }^{(4)}$. Segundo Kawakami et al, 95\% dos pacientes com úlcera duodenal apresentam $H$. pylori positivo ${ }^{(8)}$. O quadro clínico é variável de acordo com o tipo de úlcera e, principalmente, com a idade. Os sintomas mais comuns são: dor abdominal inespecífica (devido à dificuldade de caracterização nessa faixa etária), náuseas, vômitos e eructação. A hemorragia digestiva alta (HDA) é a complicação mais comum da úlcera duodenal e a anemia aparece como consequência ${ }^{(8)}$, raramente como sintoma da úlcera. A dor abdominal recorrente funcional, tão comum em pediatria, pode apresentar características semelhantes às da úlcera duodenal.

O diagnóstico de úlcera péptica pode ser feito por EDA, mesmo em crianças muito pequenas ${ }^{(9)}$. Apesar do alto custo, a endoscopia permite estabelecer o diagnóstico, o local da úlcera e a obtenção de fragmento para estudo histopatológico e pesquisa de $H$. pylori. A infecção pelo $H$. pylori pode ser diagnosticada por meio de cultura e teste da urease durante a endoscopia. Nesse teste, um fragmento da biópsia é colocado em um meio ágar com ureia e a bactéria, se presente, é capaz de hidrolisar a ureia em amônia e dióxido de carbono. Além disso, o diagnóstico da infecção pode ser feito por histologia (estudo direto da bactéria em fragmento de biópsia), reação em cadeia da polimerase, sorologia, mais utilizada em estudos epidemiológicos, e teste respiratório com ureia marcada com carbono-13 para controle de erradicação após tratamento. No caso, foi utilizado o teste da urease e a pesquisa no fragmento histopatólogico, ambos com resultado positivo.

O tratamento da doença objetiva aliviar os sintoma e cicatrizar a lesão. Está indicado para todas as crianças com doença em atividade (recorrente, cicatrizada ou complicada) 
e $H$. pylori positivas. O esquema terapêutico mais utilizado é a associação de inibidores da bomba de prótons a dois antimicrobianos $^{(6)}$. A combinação depende das cepas resistentes aos medicamentos utilizados, que varia de acordo com a sensibilidade microbiana de cada localidade. Não existe um esquema ideal, pois poucos trabalhos são realizados com crianças; o mais utilizado inclui omeprazol $(0,7$ a $1,2 \mathrm{mg} / \mathrm{kg} /$ dia) associado à claritromicina (15 a 30mg/kg/dia) e amoxicilina $(60 \mathrm{mg} / \mathrm{kg} / \mathrm{dia})$ ou metronidazol (20 a $30 \mathrm{mg} / \mathrm{kg} / \mathrm{dia}$ ) por uma ou duas semanas. Porém, essa associação tem custo elevado, havendo alguns esquemas alternativos de custo mais acessível, por exemplo, o uso de bismuto associado a dois antimicrobianos, um dos quais a furazolidona. Estudo de Kawakami et a ${ }^{(10)}$ com omeperazol, claritromicina e fura- zolidona mostrou-se tão eficaz quanto o primeiro esquema, em crianças com úlcera duodenal. Já o bismuto em terapia tripla, utilizado em um estudo europeu (PERTH), foi mais eficaz do que a terapia com os de inibidores da bomba de prótons $^{(11)}$. Após o tratamento, recomenda-se o controle da erradicação do $H$. pylori, além de manutenção caso o paciente mantenha-se sintomático.

$\mathrm{O}$ caso apresentado refere-se a um paciente com queixa frequente em pronto-socorro, a dor abdominal, porém acompanhada de anemia, o que não é característico das principais hipóteses diagnósticas para dor abdominal. Isso conduziu a uma investigação mais minuciosa, levando ao diagnóstico não habitual de úlcera péptica primária, em Serviço de Emergência Pediátrica.

\section{Referências bibliográficas}

1. Mattos S, D'Elia C. Abdomen agudo. In: Barbosa AP, D'Elia C. Condutas de urgência em pediatria. Rio de Janeiro: Atheneu; 2006. p. 777-85.

2. Leung AK, Sigalet DL. Acute abdominal pain in children. Am Fam Physician 2003;67:2321-6.

3. McCollough M, Sharieff GQ. Abdominal pain in children. Pediatr Clin North Am 2006;53:107-37.

4. Carvalho AS. Peptic ulcer. J Pediatr (Rio J) 2000;76 (Suppl 2):127-34.

5. Drumm B, Rhoads JM, Stringer DA, Sherman PM, Ellis LE, Durie PR. Peptic ulcer disease in children: etiology, clinical findings, and clinical course. Pediatrics 1988;82:410-4.

6. Bittencourt PF, Rocha GA, Penna FJ, Queiroz DM. Gastroduodenal peptic ulcer and Helicobacter pylori infection in children and adolescents. J Pediatr (Rio J) 2006;82:325-34.

7. Sakita T. Endoscopy in the diagnosis of early cancer. Clin Gastroenterol $1973 ; 2: 345-60$
8. Kawakami E, Machado RS, Fonseca JA, Patrício FR. Clinical and histologica features of duodenal ulcer in children and adolescents. J Pediatr (Rio J) 2004;80:321-5.

9. Hargrove CB, Ulshen MH, Shub MD. Upper gastrointestinal endoscopy in infants: diagnostic usefulness and safety. Pediatrics 1984;74:828-31.

10. Kawakami E, Machado RS, Ogata SK, Langner M, Fukushima E, Carelli AP et al. Furazolidone-based triple therapy for $\mathrm{H}$ pylori gastritis in children. World J Gastroenterol 2006;12:5544-9.

11. Oderda G, Shcherbakov P, Bontems P, Urruzuno P, Romano C, Gottrand F et al Results from the pediatric European register for treatment of Helicobacter pylor (PERTH). Helicobacter 2007;12:150-6.

12. Unicamp. Gastrocentro [homepage on the Internet]. Centro de Diagnóstico de Doenças do Aparelho Digestivo [cited 2010 Apr 16]. Available from: www. gastrocentro.unicamp.br 